\title{
Longevidade e oviposição de Ophyra albuquerquei (Diptera, Muscidae) em condições de laboratório ${ }^{1}$
}

\author{
Rodrigo F. Krüger²; Paulo B. Ribeiro ${ }^{3}$; Claudio J. B. de Carvalho²; Francine M. Lambrecht ${ }^{3}$ \& \\ Adrise M. Nunes ${ }^{3}$ \\ 1. Contribuição n ${ }^{\circ} 1407$ do Depto de Zoologia, Universidade Federal do Paraná (UFPR). \\ 2. Depto de Zoologia, UFPR, Caixa Postal 19020, 81531-980, Curitiba, PR, Brasil. Bolsista CAPES (rfkruger@ bio.ufpr.br); bolsista \\ CNPq (cjbcarva@ufpr.br). \\ 3. Depto de Microbiologia e Parasitologia, Universidade Federal de Pelotas, Caixa Postal 354, 96010-900, Pelotas, RS, Brasil. \\ (bretanha@ufpel.tche.br).
}

\begin{abstract}
Longevity and oviposition of Ophyra albuquerquei (Diptera, Muscidae) under laboratory conditions. Aspects related to the longevity and oviposition of Ophyra albuquerquei Lopes, 1985 are studied. Males had a mean lifespan longer than females (40.24 vs. 33.15 days, respectively), while still possessing qualitative advantages during this period. Females $O$. albuquerquei were fed on powdered milk, fish flour, refined sugar and water, and provided fish flour and moistened sawdust for oviposition. The length of the oviposition period for females was 46.75 days, and most of the deposition of the eggs occurred in the first days of the colony. Females completed $50 \%$ of their egg deposition by Day 16, seven days before the large last mortality peak and about 12 days after the first oviposition in the colony. Females deposited an average of 184 eggs per individual. Mortality of males, unlike females, was low until the 28th day, and increased thereafter. It was demonstrated that it is possible to maintain colonies of this species under laboratory conditions for at least 28 days with high fertility and low cost.
\end{abstract}

KEYWORDS. Artificial diet, lifespan, mortality, Ophyra, senescence.

\section{INTRODUÇÃO}

No Brasil ocorrem cinco espécies de Ophyra Robineau-Desvoidy, 1830 (Azeliinae) (CARvalHo et al., 1993) que são freqüentemente encontradas em granjas avícolas e de suínos (Lomônaco \& Prado, 1994) e em matéria orgânica animal em decomposição, como cadáveres de mamíferos, onde ocorrem em baixa freqüência (Moura et al., 1997).

Em granjas avícolas e de suínos, as espécies deste gênero vêm assumindo projeção no controle biológico das larvas de dípteros que comumente ocorrem nestes sistemas com alta taxa de fermentação bacteriana (FARKAS et al., 1998), com destaque para Ophyra aenescens (Wiedemann, 1830), que tem sido estudada no Brasil quanto aos aspectos do desenvolvimento de imaturos e adultos (Almeida et al., 1999; Ribeiro et al., 2000a; 2000b). Nos substratos que apresentam estas características, as larvas de Ophyra ignava (Harris, 1780), O. capensis (Wiedemann,1818) e O. aenescens alimentam-se das larvas de Musca domestica L., 1758 (Muscidae, Muscinae) (Anderson \& Poorbaugh, 1964; Betke et al., 1989; Tsankova \& Luvchiev, 1993) e Stomoxys calcitrans L., 1758 (Muscinae), heleomizíneos, sarcofagídeos e califorídeos, utilizam a predação facultativa de terceiro instar como estratégia para obtenção de recursos protéicos (SKIDMORE, 1985).

Esta estratégia alimentar gerou a proposta de uma dieta para larvas de $O$. aenescens composta principalmente de farinha de carne (Youngman et al., 1991), o que facilitou sua manutenção em laboratório. HogsetTe \& WASHINGTON (1995), ao utilizarem dietas vegetais para esta espécie, não obtiveram bom desenvolvimento dos estágios imaturos, somente alcançaram sucesso na criação quando adicionaram farinha de peixe. RiBeIRo et al. (2000a) modificaram as proporções entre proteínas e amido, desenvolvendo uma dieta composta por cinco partes de farinha de peixe, três de serragem e duas de farinha de trigo, obtendo alta viabilidade entre os imaturos desta espécie. Para os adultos, Youngman et al. (1991) forneceram uma dieta composta por uma parte de farinha de carne misturada com duas partes de leite em pó e duas de açúcar; RIBEIRO et al. (2000b) seguiram a mesma dieta utilizando farinha de peixe no lugar de farinha de carne.

Apesar do conhecimento sobre a capacidade de controle das espécies de Ophyra sobre larvas de outras espécies de Diptera, sua utilização no manejo ecológico de pragas tem esbarrado no fato de que elas podem veicular enterovírus, enterobactérias e asquelmintos (Greenberg, 1971). Contudo, ocorrem com baixa freqüência no ambiente (RiBEIRo et al., 2000c), preferindo áreas rurais ou de florestas (COSTA et al., 2000), devido à necessidade de locais de luz restrita, próximos à vegetação para congregação e acasalamento; não se propagam para residências e, por isso, não apresentam importância como veiculadores de patógenos (Nolam III \& Kissam, 1987).

A biologia de Ophyra albuquerquei Lopes, 1985 é desconhecida, sabendo-se apenas que tem modelo de flutuação populacional semelhante ao de $O$. aenescens, o que infere a possibilidade de possuírem exigências biológicas semelhantes (Ribeiro et al., 2000c). O estudo da longevidade e oviposição em laboratório é o princípio do entendimento da bionomia de $O$. albuquerquei, porque a longevidade, assim como a mortalidade, estão fortemente correlacionadas com aspectos reprodutivos, gerando custos fisiológicos que têm papel determinante na história de vida de uma espécie (REzNICK, 1985).

Para esclarecimento de dados bionômicos são 
necessárias criações em laboratório que disponibilizem informações sobre parâmetros de difícil avaliação no campo. É necessária a utilização de uma faixa de temperatura que permita a comparação com os dados estimados para outras espécies. Para isto, a faixa de desenvolvimento mais aconselhável é aquela na qual os insetos possuem desenvolvimento mais rápido com maior número de descendentes, entre os limites de $15^{\circ} \mathrm{C}$ e $38^{\circ} \mathrm{C}$, sendo que a temperatura de $25^{\circ}$ é chamada de ponto ótimo ou ponto de desenvolvimento (SiLveira-Neto et al., 1976).

Considerando a longevidade e a oviposição como os dois fatores que mais influenciam a dinâmica de uma população, e que $O$. albuquerquei pode ser utilizada em programa de controle de $M$. domestica, objetiva-se estimar a longevidade e capacidade de oviposição dessa espécie em laboratório, observando a intensidade destes fatores no decorrer do tempo de vida, como os períodos de pré-oviposição, oviposição e pós-oviposição.

\section{MATERIAL E MÉTODOS}

Em fevereiro de 2001 foi estabelecida uma colônia de $O$. albuquerquei em laboratório, em câmara climatizada, a partir de adultos capturados em granja avícola do Campus da Universidade Federal de Pelotas (UFPel), Rio Grande do Sul (3145'48' S, 52²9'02'”W), para obtenção de ovos, larvas e pupários. Os adultos

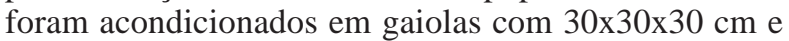
alimentados com leite em pó, açúcar refinado e farinha de peixe, numa proporção de 2:2:1 respectivamente, oferecendo-se água em pequenos frascos com espuma de poliestireno cobrindo a superfície do líquido, conforme RiBEIRo et al. (2000b). Ração e água foram oferecidas ad libitum. A colônia foi mantida em temperatura média de $25^{\circ}$, variando entre $20^{\circ}$ e $30^{\circ} \mathrm{C}$, umidade relativa do ar de $75 \%$, variando entre $65 \%$ e $85 \%$, e fotoperíodo de 12 horas. As larvas foram alimentadas com meio de cultura composto de farinha de peixe e de serragem em uma proporção de 1:1, adicionando-se água até tornar o meio pastoso. A renovação da colônia foi realizada a partir de posturas da própria colônia. As pupas foram incubadas a $26^{\circ} \mathrm{C} \pm 1^{\circ} \mathrm{C}$ em serradura úmida até a emergência dos adultos. Estes receberam a mesma dieta de O. aenescens, conforme RIBEIRO et al. (2000b).

Para estimar o período de pré-oviposição, oviposição, pós-oviposição, tempo letal médio, capacidade de postura e longevidade dos adultos, foram mantidas uma, três, e quatro colônias oriundas de 70, 60 e 50 pupas respectivamente, totalizando 450 pupas, coletadas aleatoriamente da quarta geração mantida em laboratório. As colônias foram observadas diariamente em intervalos de 24 horas para remoção dos insetos mortos e das posturas e a sexagem realizada logo após a morte dos exemplares. A razão sexual foi obtida pela proporção entre o número de fêmeas e a soma do número de fêmeas e machos, conforme SilVEIRA-NETO et al. (1976).

Foram realizados teste de qui-quadrado para razão sexual em cada colônia a 0,05 de probabilidade, considerando 0,5 a razão normal, e teste t para comparar a longevidade média de machos e fêmeas no nível de 0,05 de probabilidade.

Espécimes-testemunho que emergiram da criação em laboratório foram depositados na Coleção de
Entomologia Pe. Jesus Santiago Moure, Departamento de Zoologia, Universidade Federal do Paraná, Curitiba e Coleção de Insetos de Importância Médica e Veterinária, Departamento de Microbiologia e Parasitologia, Universidade Federal de Pelotas, Pelotas.

\section{RESULTADOS}

A longevidade média dos machos das oito colônias de $O$. albuquerquei foi de 40,24 $\pm 14,51$ dias, diferenciandose estatisticamente da média das fêmeas de 33,15 $\pm 17,01$ dias; a mínima dos machos variou de três a 29 dias e a das fêmeas de cinco a 14 dias. A máxima dos machos oscilou de 50 a 74 dias e das fêmeas de 51 a 70 dias. O tempo letal médio das colônias foi de $39 \pm 8,07$ dias, variando de 24 a 49 dias (tab. I).

Tabela I. Longevidade e tempo letal médio de Ophyra albuquerquei Lopes, 1985, em condições de laboratório (COL, colônias; M, machos; F, fêmeas; TLM, tempo letal-médio da colônia; $\bar{x}$, média; s, desvio padrão; $\Delta$, variação; médias seguidas de letras diferentes diferem significativamente $(\mathrm{p}=0,05)$ pelo teste t).

\begin{tabular}{|c|c|c|c|c|c|}
\hline \multirow[t]{2}{*}{$\mathrm{COL}$} & \multicolumn{4}{|c|}{ Longevidade (dias) } & \multirow[t]{2}{*}{ TLM (dias) } \\
\hline & M & $\overline{\mathrm{x}} \mathrm{M}$ & $\mathrm{F}$ & $\bar{x} F$ & \\
\hline 01 & $18-72$ & 45,75 & $06-53$ & 29,40 & 44 \\
\hline 02 & $03-50$ & 30,18 & $05-51$ & 25,81 & 24 \\
\hline 03 & $06-58$ & 31,90 & $08-70$ & 31,96 & 35 \\
\hline 04 & $06-74$ & 41,17 & $14-67$ & 36,42 & 43 \\
\hline 05 & $14-59$ & 43,43 & $07-59$ & 38,71 & 46 \\
\hline 06 & $27-58$ & 42,90 & $13-58$ & 32,23 & 35 \\
\hline 07 & $21-52$ & 36,35 & $10-64$ & 34,79 & 36 \\
\hline 08 & $29-67$ & 50,54 & $13-66$ & 39,68 & 49 \\
\hline$\Delta$ & $03-74$ & $30,18-50,54$ & $05-70$ & , $81-39$, &, $6824-49$ \\
\hline $\bar{x}+s$ & 40,24 & $\pm 14,51^{\mathrm{a}}$ & 33,15 & $17,01^{\mathrm{b}}$ & $39,00 \pm 8,07$ \\
\hline
\end{tabular}

A mortalidade de machos foi reduzida até o $28^{\circ}$ dia, com exceção do $16^{\circ}$ dia em que houve um pico, sendo a freqüência relativa acumulada menor que $25 \%$. Após o $28^{\circ}$ dia registrou-se um incremento neste parâmetro, ocorrendo sucessivos picos que culminaram em um acme, compreendido pelos $57^{\circ}$ e $58^{\circ}$ dias juntos. Somente entre o $35^{\circ}$ e $56^{\circ}$ dias ocorreu a mortalidade de aproximadamente $40 \%$ dos machos, atingindo freqüência relativa acumulada de $50 \%$ no $42^{\circ}$ dia (fig. 1).

As fêmeas tiveram até o $28^{\circ}$ dia os principais picos de mortalidade que constituíram dois acmes, um compreendido entre o $12^{\circ}$ e o $16^{\circ}$ dias e outro entre o $21^{\circ}$ e o $25^{\circ}$ dias. Nestes dois períodos, $40 \%$ das fêmeas morreram. Somente no $22^{\circ}$ e $23^{\circ}$ dias ocorreu mortalidade de $13,43 \%$. A partir do $28^{\circ}$ dia, houve diminuição da frequiência absoluta diária da mortalidade de fêmeas, acontecendo um pico de oito mortes no $36^{\circ}$ e dois picos de seis mortes, um no $37^{\circ}$ e outro no $58^{\circ}$ dia. Até o $32^{\circ}$ dia, a mortalidade atingiu $50 \%$, sendo que até o $52^{\circ}$ dia, $75 \%$ dos espécimes morreram (fig. 2).

Entre os adultos, ocorreram cinco picos com freqüência acima de 12 mortes que correspondem ao $16^{\circ}$, $22^{\circ}, 23^{\circ}, 38^{\circ}$ e $58^{\circ}$ dias. Cerca de $35 \%$ dos adultos morreram até o $28^{\circ}$ dia, aumentando para $50 \%$ até o $37^{\circ}$ dia. No $50^{\circ}$ dia, a freqüência relativa acumulada da mortalidade atingiu $75 \%$ (fig. 3). 

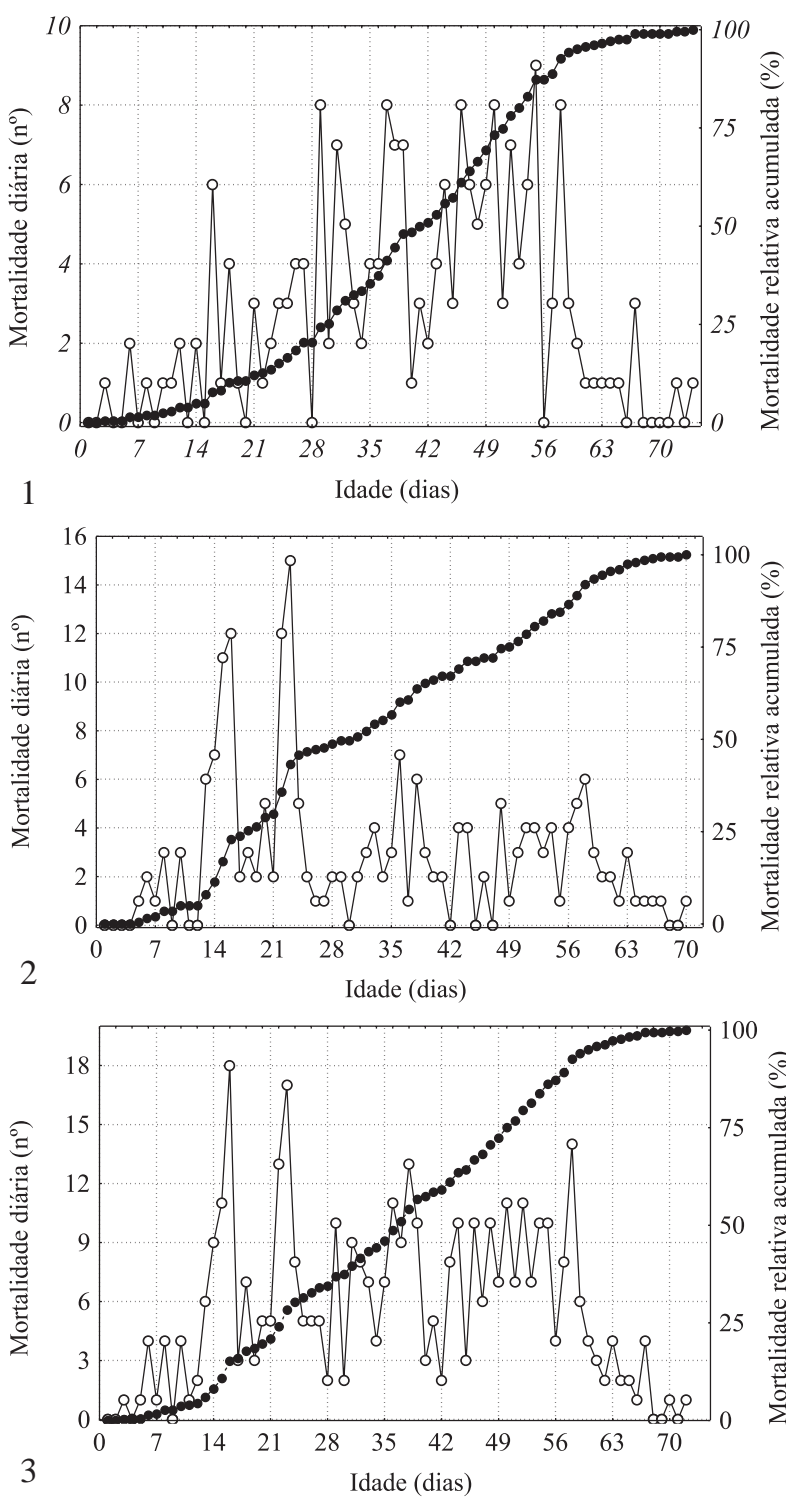

Figs. 1-3. Freqüência da mortalidade de Ophyra albuquerquei Lopes, 1985, em função da idade, em condições de laboratório: 1, machos; 2 , fêmeas; 3 , adultos $(-\infty$, mortalidade diária; $\rightarrow$, mortalidade relativa acumulada).

O período médio de pré-oviposição de $O$. albuquerquei foi de 4,63 $\pm 1,19$ dias com variação de três a sete dias. A oviposição durou em média 46,75 $\pm 8,99$ dias variando de 31 a 59 dias. A pós-oviposição, período compreendido entre a última oviposição e a morte da última fêmea, foi de 9,63 $\pm 8,11$ dias em média, oscilando de dois a 25 dias (tab. II).

O número de ovos por fêmea foi de 184,18 \pm 45 , considerando-se as oito colônias. Esta média para cada colônia variou de 123,81 a 236,76 ovos por fêmea (tab. III). Até o $28^{\circ}$ dia, $75 \%$ dos ovos foram colocados no substrato, sendo que entre o $5^{\circ}$ e o $21^{\circ}$ dia houve a oviposição de $60 \%$ dos ovos. Os três principais picos ocorreram no $6^{\circ}, 8^{\circ}$ e $16^{\circ}$ dias. A partir do $28^{\circ}$ dia registraram-se apenas dois picos de oviposição acima de 1000 ovos por dia, um no $31^{\circ}$ e outro no $38^{\circ}$ dia. Até este período, em torno de $93 \%$ dos ovos foram depositados no substrato, sendo necessários mais 28 dias para a oviposição de apenas $7 \%$ do total de ovos produzidos por O. albuquerquei (fig. 4).

A razão sexual foi de 0,49 considerando-se os 406 adultos, variando de 0,35 a 0,60 para as oito colônias. Pelo teste de qui-quadrado houve diferença significativa entre o número de machos e fêmeas nas colônias 01 ( $p$ < $0,04)$ e $05(p<0,05)$, o que corresponde a dois machos para cada fêmea (tab. III).

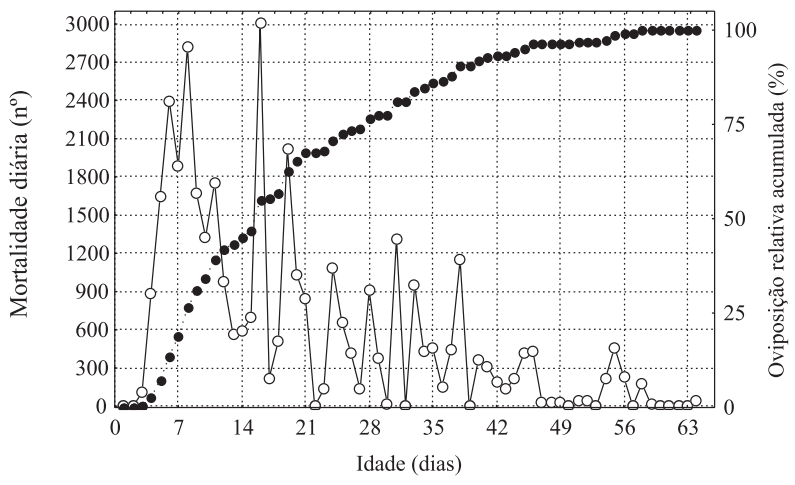

Fig. 4. Freqüência da oviposição de Ophyra albuquerquei Lopes, 1985, em função da idade, em condições de laboratório (- - , oviposição diária; $\bullet$, oviposição relativa acumulada).

Tabela II. Períodos de pré-oviposição, oviposição e pósoviposição de Ophyra albuquerquei Lopes, 1985, em condições de laboratório (COL, colônias; PrO, pré-oviposição; Ov, oviposição; PO, pós-oviposição; *, período estimado entre a última oviposição e a morte da última fêmea; $\Delta$, variação; $\bar{x}$, média; s, desvio padrão).

\begin{tabular}{cccc}
\hline COL & PrO (dias) & Ov (dias) & PO (dias)* \\
\hline 01 & 03 & 31 & 19 \\
02 & 05 & 41 & 02 \\
03 & 04 & 41 & 08 \\
04 & 05 & 54 & 03 \\
05 & 07 & 45 & 07 \\
06 & 04 & 51 & 08 \\
07 & 04 & 52 & 25 \\
08 & 05 & 59 & 05 \\
\hline$\Delta$ & $03-07$ & $31-59$ & $02-25$ \\
$\overline{\mathrm{x}}+\mathrm{s}$ & $4,63 \pm 1,19$ & $46,75 \pm 8,99$ & $9,63 \pm 8,11$ \\
\hline
\end{tabular}

Tabela III. Capacidade de postura e razão sexual de Ophyra albuquerquei Lopes, 1985, em condições de laboratório (COL, colônias; M, machos; F, fêmeas; $\Sigma$, somatório; PT, postura total; $\mathrm{O}$, ovos; RS, razão sexual; $\overline{\mathrm{x}}$, média; s, desvio padrão; $\mathrm{x}^{2}$, quiquadrado; ns, não significativo; T, total).

\begin{tabular}{|c|c|c|c|c|c|c|c|}
\hline \multirow[b]{2}{*}{$\mathrm{COL}$} & \multicolumn{3}{|c|}{ Espécimes } & \multirow[b]{2}{*}{$\mathrm{PT}$} & \multirow[b]{2}{*}{$\mathrm{O} / \mathrm{F}$} & \multirow[b]{2}{*}{$\mathrm{RS}$} & \multirow[b]{2}{*}{$\mathrm{x}^{2}$} \\
\hline & $\mathrm{M}$ & $\mathrm{F}$ & $\Sigma$ & & & & \\
\hline 01 & 28 & 15 & 43 & 3239 & 215,93 & 0,35 & 0,04 \\
\hline 02 & 33 & 37 & 70 & 5691 & 172,45 & 0,53 & $\mathrm{~ns}$ \\
\hline 03 & 21 & 25 & 46 & 5919 & 236,76 & 0,54 & $\mathrm{~ns}$ \\
\hline 04 & 24 & 19 & 43 & 2720 & 143,16 & 0,44 & $\mathrm{~ns}$ \\
\hline 05 & 30 & 17 & 47 & 3168 & 186,35 & 0,36 & 0,05 \\
\hline 06 & 21 & 31 & 52 & 3931 & 123,81 & 0,60 & $\mathrm{~ns}$ \\
\hline 07 & 23 & 29 & 52 & 5195 & 179,14 & 0,56 & $\mathrm{~ns}$ \\
\hline 08 & 26 & 28 & 54 & 7157 & 255,61 & 0,52 & $\mathrm{~ns}$ \\
\hline $\mathrm{T}$ & 206 & 201 & 407 & 37020 & & & \\
\hline $\bar{x}+s$ & & & & & $18 \pm 45,00$ & 0,49 & \\
\hline
\end{tabular}




\section{DISCUSSÃO}

Nas condições de criação de $O$. albuquerquei, tanto machos quanto fêmeas foram mais longevos do que machos e fêmeas de $O$. aenescens, cuja longevidade média foi de 19,7 e 21,3 dias respectivamente, não apresentando diferença significativa entre estas médias (RibeIRo et al., 2000b).

Em dietas com base protéica como carne e outros derivados, os muscídeos que são carnívoros facultativos, especialmente aqueles de Azeliinae, tendem a exibir comportamento semelhante quanto à utilização do substrato e história de vida. Em Synthesiomyia nudiseta (Wulp, 1883) (Azeliinae) criada em carne bovina e açúcar (RABINOVICH, 1970) e também em fígado cru, açúcar e leite em pó (Almeida \& Almeida, 1996), os machos sobrevivem em média 34 a 49 dias e as fêmeas 31 a 41 dias, aproximando-se das médias verificadas para $O$. albuquerquei. A necessidade de maior tempo de vida para os machos destas espécies pode ser devido ao fato de que nem todos estão aptos para reprodução nas primeiras semanas após a emergência, provavelmente porque nesta subfamília machos dominantes ocorram com maior frequiência, o que faz com que mais indivíduos prolonguem o seu tempo de vida.

O período em que ocorre a maior oviposição de $O$. albuquerquei coincide com o de $O$. aenescens, entre o $8^{\circ}$ e o $16^{\circ}$ dias (RIBEIRo et al., 2000b), o que indica que possuem modelo de oviposição semelhante, independente do substrato de oviposição utilizado. $\mathrm{O}$ que torna estas duas espécies diferentes quanto a oviposição é a frequiência relativa acumulada por período. Até o $28^{\circ}$ dia mais de $80 \%$ da mortalidade de $O$. aenescens ocorreu, sendo ovipositados mais de $90 \%$ dos ovos (RIBEIRO et al., 2000b). Para este mesmo período, as fêmeas de $O$. albuquerquei depositaram no substrato $75 \%$ dos ovos e a freqüência relativa acumulada da mortalidade de adultos atingiu 35\%. Esta comparação demonstra que O. aenescens, em laboratório, cumpre a função reprodutiva muito antes de $O$. albuquerquei, que possui capacidade de oviposição média muito inferior ao daquela espécie, e de O. ignava, 285 e 300 ovos por fêmea, respectivamente (Axtell, 1986; Ribeiro et al., 2000b). A capacidade de oviposição de O. albuquerquei é semelhante a de fêmeas de $O$. aenescens provenientes da Alemanha (Stein et al., 1976 apud SKIDMORE, 1985) e de Muscina stabulans (Fallén, 1817) (Azeliinae) (Séguy, 1923 apud SKIDMORE, 1985).

Tanto $O$. albuquerquei quanto $O$. aenescens possuem potencial reprodutivo inferior ao de $M$. domestica, uma presa em potencial destas duas espécies, que em média pode colocar até 494,9 ovos por fêmea a $30^{\circ} \mathrm{C} \mathrm{e}$, com temperatura de $25^{\circ} \mathrm{C}$, uma fêmea desta espécie ovipõe em média 433 ovos (Fletcher et al., 1990). Sendo uma presa com diversos inimigos naturais, esta espécie tem que ter potencial reprodutivo muito maior do que seus inimigos, logo a soltura massal de pupas de $O$. albuquerquei seria a estratégia mais aconselhável para sobrepor a capacidade reprodutiva de $M$. domestica no ambiente.

As variações no número de ovos por fêmea podem ter várias origens, desde a dieta para os adultos quanto à densidade em uma gaiola ou mesmo a estratégia de reprodução inerente à espécie. A fecundidade é um atributo que pode variar com a densidade larval, e caso ocorram densidades elevadas entre as larvas, os adultos sofrem com a diminuição do tamanho, afetando a capacidade de oviposição das fêmeas. Isto foi verificado em Chrysomya putoria (Wiedemann, 1830) (Calliphoridae) (RIBEIRO, O. B. et al., 1993) e M. domestica (RIBEIRo et al., 1995), o que não ocorreu com O. albuquerquei, pois em média, foi mantida uma larva por grama no meio de criação desta espécie.

A $25^{\circ} \mathrm{C}$ as fêmeas de $M$. domestica levam 18,1 dias em média para depositar $50 \%$ da totalidade de ovos (FLETCHER et al., 1990), sendo uma estimativa muito aproximada de $O$. albuquerquei que deposita $50 \%$ dos ovos até o $16^{\circ}$ dia (fig. 4), havendo portanto sincronia entre os principais períodos de oviposição destas duas espécies.

A reprodução em $O$. albuquerquei pode gerar algum custo fisiológico para as fêmeas, pois os picos de mortalidade foram em seguida aos de oviposição, $6^{\circ}, 8^{\circ}$ e $16^{\circ}$ dias, sendo os intervalos entre um de mortalidade e um de oviposição de seis a oito dias. Para machos, esta relação é menos evidente, já que houve apenas um pico de mortalidade no período de maior oviposição. Custo fisiológico pode ser verificado em Fannia pusio (Wiedemann, 1830) (Diptera, Fanniidae) que possui relação inversamente proporcional entre a longevidade média das fêmeas e a fecundidade nas temperaturas de 20 e $27^{\circ} \mathrm{C}$ (Marchiori \& Prado, 1995).

Poucos machos de O. albuquerquei devem estar relacionados diretamente à produção de descendentes nas primeiras semanas da colônia, sendo que a maioria deles e menor parcela de fêmeas podem ter uma estratégia de espera. Isto ocorre com fêmeas de Ceratitis capitata (Wiedemann, 1824) (Diptera, Tephritidae), que são capazes de realizar dois modos distintos de estratégia reprodutiva: um de espera, com baixa mortalidade, no qual são produzidos poucos ovos ou nenhum; e outro reprodutivo, com produção de descendentes, onde há decréscimo nesta função, enquanto a mortalidade inicial que era baixa se torna maior no decorrer do tempo, processo denominado de senescência (CAREY et al., 1998). Este processo pode ser observado nas trajetórias da freqüência relativa acumulada da oviposição e da mortalidade de $O$. albuquerquei. Considerando estas duas trajetórias, a taxa reprodutiva reduz no decorrer do tempo e a mortalidade aumenta.

Organismos tipicamente de vida longa começam a reproduzir em idades mais avançadas do que os de vida curta. Isto não ocorre com as colônias de $O$. albuquerquei, que apesar de terem longevidade média muito superior à de $O$. aenescens, possuem período de pré-oviposição semelhantes, cerca de quatro (JoHnSON \& Venard, 1957) a 5,33 dias (Ribeiro et al., 2000b). O período de pré-oviposição estimado não leva em consideração que as fêmeas de $O$. albuquerquei provavelmente não tenham o mesmo período para o ciclo gonadotrófico, uma vez que a avaliação desta estimativa reprodutiva levou em consideração as colônias como um todo, não avaliando casais separados uns dos outros. Em S. nudiseta, a $20-21^{\circ} \mathrm{C}$, a pré-oviposição pode ser de 
10,3 \pm 0,3 dias quando criada em colônia (RABINOvich, 1970) ou de 15,5 dias quando criada em casais separados com alto desvio padrão (AlmeIDA et al., 1997). Estes dois períodos são superiores ao de $O$. albuquerquei, apesar desta espécie possuir longevidade média próxima à de $S$. nudiseta. Este período também pode variar de acordo com a dieta utilizada. Em Muscina stabulans, foi observado que variou de seis dias em dietas com fígado até 12,6 dias em ração de aves (Tirone et al., 1996).

O período de oviposição de $O$. albuquerquei aproxima-se do estimado para $S$. nudiseta a $28^{\circ} \mathrm{C}$, que foi de 52 dias (RABINOvich, 1970) e o de pós-oviposição apresentou grande variação, sendo semelhante ao de Sarconesia chlorogaster (Wiedemann, 1830) (Calliphoridae, Toxotarsinae) (BоnAтto, 1996), principalmente quanto ao desvio padrão desta estimativa.

A oviposição pode ter sido prolongada devido à estratégia de espera que poucas fêmeas assumem. Já a pós-oviposição possui grande variação porque as fêmeas que não ovipositaram tiveram sua longevidade prolongada, uma vez que não foram expostas ao desgaste fisiológico da reprodução (REZnick, 1985). A taxa de fêmeas que não fazem oviposição é extremamente variável. Em Dermatobia hominis (Linnaeus, 1781) (Oestridae), até $43 \%$ das fêmeas não realizam postura (RIBEIRO, P. B. et al., 1993). Esta taxa é de 10\% em Sarconesia chlorogaster (BonatTo, 1996) e de apenas 2,5\% em Synthesiomyia nudiseta (AlmeIDA et al., 1997).

A razão sexual foi de aproximadamente 0,5 , sendo semelhante à de O. aenescens (RIBEIRO et al., 2000b), não demonstrando uma tendência em relação a um dos dois sexos, com exceção das colônias que tiveram a terceira e a quarta maior capacidade de oviposição entre as oito colônias.

Testes em laboratório com moscas criadas artificialmente impõem sérias restrições às teorias elaboradas sobre os resultados obtidos, pois as populações mantidas sob condições artificiais foram adaptadas àquela situação. Uma população em ambiente natural pode não se comportar da mesma maneira, o que gera a necessidade da confrontação entre os dados obtidos em sistemas artificiais e aqueles que podem ser obtidos no ambiente.

Em laboratório, com a finalidade de manutenção de colônias de $O$. albuquerquei para programas de manejo, a baixo custo de produção e facilidade de manuseio, devese manter a colônia até o $28^{\circ}$ dia, 11 dias antes do tempo letal médio. Até este período ocorre a produção da imensa maioria dos ovos, o que viabiliza a manutenção de aproximadamente 13 gerações por ano em laboratório. Ao interromper o desenvolvimento neste período, onde possivelmente haja custos fisiológicos envolvidos e onde a competição por acasalamentos é mais intensa, poderá acontecer modificação da mortalidade por idade específica, alterando o ajustamento da população mantida nestas condições, gerando seleção da prole daqueles indivíduos que se reproduzem precocemente.

Conclui-se que existe processo de senescência entre os adultos, principalmente nas fêmeas, e que é possível manter $O$. albuquerquei em condições artificiais, sendo a dieta oferecida apropriada para sua criação.
Agradecimentos. Ao CNPq, pela bolsa de Mestrado (Processo 130415/00-6) do autor sênior e bolsa PIBIC de F. M. Lambrecht; ao médico-veterinário Paulo R. P. Costa (in memorian), pelo auxílio na manutenção das colônias em laboratório; ao Dr. Phillip Scholl (University of Nebraska), pelas sugestões para o abstract. À comissão editorial pelas valiosas críticas, sugestões e contribuições.

\section{REFERÊNCIAS BIBLIOGRÁFICAS}

Almeida, J. M. D' \& Almeida, J. R. DE. 1996. Longevidade e curva de sobrevivência de oito espécies de dípteros caliptrados (Calliphoridae, Muscidae e Sarcophagidae), em condições de laboratório. Revista Brasileira de Biologia, Rio de Janeiro, 56(3):497-505.

Almeida, J. M. D’; Borges, C. \& Gonçalves, C. A. 1999. Desenvolvimento pós-embrionário de Ophyra aenescens (Wiedemann, 1830) (Diptera: Muscidae) em diferentes dietas, sob condições de laboratório. Memórias do Instituto Oswaldo Cruz, Rio de Janeiro, 94(1):123126.

Almeida, J. M. D'; Piana, M. L. G. \& Selem, C. T. 1997. Comportamento reprodutivo de Synthesiomyia nudiseta van der Wulp (Diptera: Muscidae) sob condições de laboratório. Memórias do Instituto Oswaldo Cruz, Rio de Janeiro, 92(4):563, 564.

Anderson, J. R. \& Poorbaugh, J. H. 1964. Biological control possibility for house flies. California Agriculture, Los Angeles, 18(9):2-4.

Axtell, R. C. 1986. Fly control in confined livestock and poultry production. Greensboro, CIBA-GEIGY. 59p.

BetKe, P.; Hiepe, T. et al. 1989. Biological control of Musca domestica with Ophyra aenescens on pig production enterprises. Monastshefte für Veterinarmedizin, Berlin, 44:842-844.

Bonatto, S. R. 1996. Ciclo de vida de Sarconesia chlorogaster (Wiedemann) (Diptera, Calliphoridae, Toxotarsinae), criada sob condições de laboratório em dieta artificial. Revista Brasileira de Zoologia, Curitiba, 13(3):685706.

Carey, J. R.; Liedo, P. et al. 1998. Dual modes of aging in mediterranean fruit fly females. Science, Washington, 281:996-998.

Carvalho, C. J. B. De; Pont, A. C. et al. 1993. Part II. Muscidae. In: Carvalho, C. J. B. DE. ed. A catalogue of the Fanniidae and Muscidae (Diptera) of the Neotropical Region. São Paulo, Sociedade Brasileira de Entomologia. p. 1-201.

Costa, P. R. P.; Franz, R. L. et al. 2000. Synanthropy of Ophyra spp. (Diptera, Muscidae) in Pelotas, RS, Brazil. Revista Brasileira de Parasitologia Veterinária, Rio de Janeiro, 9(2):165-168.

Farkas, R.; Hogsette, J. A. \& Börzsönyi, L. 1998. Development of Hydrotaea aenescens and Musca domestica (Diptera: Muscidae) in poultry and pig manures of different moisture content. Environmental Entomology, Lanham, 27(3):695699.

Fletcher, M. G.; Axtell, R. C. \& Stinner, R. E. 1990. Longevity and fecundity of Musca domestica (Diptera, Muscidae) as a function of temperature. Journal of Medical Entomology, Lanham, 27(5):922-926.

Greenberg, B. 1971. Flies and disease. Ecology, classification and biotic associations. Princeton, Princeton University. 586p.

Hogsette, J. A. \& Washington, F. 1995. Quantitative mass production of Hydrotaea aenescens (Diptera: Muscidae). Journal of Economic Entomology, Lanham, 88(5):12381242 .

Johnson, W. T. \& Venard, C. E. 1957. Observations on the biology and morphology of Ophyra aenescens (Diptera: Muscidae). Ohio Journal of Science, Columbus, 57(1):2126.

Lomônaco, C. \& Prado, A. P. 1994. Estrutura comunitária e dinâmica populacional da fauna de dípteros e seus inimigos naturais em granjas avícolas. Anais da Sociedade 
Entomológica do Brasil, Londrina, 23(1):71-80.

Marchiori, C. H. \& Prado, A. P. 1995. Longevidade e fecundidade de Fannia pusio (Wiedemann, 1830) (Diptera: Fanniidae) em laboratório. Revista Brasileira de Biologia, Rio de Janeiro, 55(4):571-575.

Moura, M. O.; Carvalho, C. J. B. De \& Monteiro-Filho, E. L. A 1997. A preliminary analysis of insects of medico-legal importance in Curitiba, State of Paraná. Memórias do Instituto Oswaldo Cruz, Rio de Janeiro, 92(2):269274.

Nolam III, M. P. \& Kissam, J. B. 1987. Nuisance potential of a dump fly, Ophyra aenescens (Diptera: Muscidae), breeding at poultry farms. Environmental Entomology, Lanham, 16(3):828-831.

Rabinovich, J. 1970. Vital statistics of Synthesiomyia nudiseta (Diptera: Muscidae). Annals of the Entomological Society of America, Lanham, 63(3):749-752.

REZNICK, D. 1985. Costs of reproduction: an evaluation of the empirical evidence. Oikos, Copenhagen, 44:257-267.

Ribeiro, O. B.; Prado, A. P. \& Guimarães, J. H. 1993. Competição intra-específica em Chrysomya putoria (Wiedemann, 1830) (Diptera, Calliphoridae) em meio artificial. Revista Brasileira de Entomologia, São Paulo, 37(4):641-652.

Ribeiro, P. B.; Carvalho, C. J. B. De et al. 2000 a. Desenvolvimento de Ophyra aenescens Wiedemann, 1830 (Diptera, Muscidae, Azeliinae), em diferentes temperaturas, em condições de laboratório. Revista Brasileira de Agrociência, Pelotas, 6(1):80-87.

2000b. Longevidade, oviposição e viabilidade pupal de Ophyra aenescens Wiedemann, 1830 (Diptera, Muscidae,
Azeliinae), em condições de laboratório. Revista Brasileira de Agrociência, Pelotas, 6(3):264-268.

2000c. Flutuação populacional das espécies de Ophyra Robineau-Desvoidy (Diptera, Muscidae, Azeliinae) na região de Pelotas, RS. Arquivos do Instituto Biológico, São Paulo, 67(2):205-214.

Ribeiro, P. B.; Vianna, E. E. S. et al. 1993. Período de vida e capacidade de postura da Dermatobia hominis, em laboratório. Revista Brasileira de Parasitologia Veterinária, Rio de Janeiro, 2(1):29-31.

Ribeiro, S. C.; Souza, A. M. de \& Lomônaco, C. 1995. Influência de fatores ambientais na determinação do tamanho de Musca domestica L. (Diptera: Muscidae). Revista Brasileira de Biologia, Rio de Janeiro, 55(4):633-637.

Silveira-Neto, S.; NaKano, O. et al. 1976. Manual de ecologia dos insetos. São Paulo, Ceres. 419p.

Skidmore, P. 1985. The biology of the Muscidae of the world. Dordrecht, Dordrecht Kunk. 550p.

Tirone, G.; Parise, P. P. \& Avancini, R. M. P. 1996. Ovarian development in Muscina stabulans (Diptera, Muscidae) on differents diets. Revista Brasileira de Entomologia, São Paulo, 40(1):105, 106

Tsankova, R. N. \& Luvchiev, V. I. 1993. Laboratory investigations on the larval zoophagy of Ophyra capensis - an antagonist of Musca domestica. Applied Parasitology, Berlin, 34(3):221-228.

Youngman, R. R.; Turner, E. C., JR. \& Reuzler, P. L. 1991. Instructions on insectary establishment, mass rearing, and release of Ophyra aenescens: A house fly predator. Virginia, Virginia Cooperative Extension. n. $444-769,4 \mathrm{p}$ 